\title{
Optical manipulation of the exciton charge state in single layer tungsten disulfide
}

\author{
A. A. Mitioglu ${ }^{1,2}$ P. Plochocka, ${ }^{1}$ J. N. Jadczak,${ }^{1,3}$ W. Escoffier, ${ }^{1}$ G. L. J. A. Rikken, ${ }^{1}$ L. Kulyuk,${ }^{2}$ and D. K. Maude ${ }^{1}$ \\ ${ }^{1}$ Laboratoire National des Champs Magnétiques Intenses, \\ UPR 3228, CNRS-UJF-UPS-INSA, Grenoble and Toulouse, France \\ ${ }^{2}$ Institute of Applied Physics, Academiei Str. 5, Chisinau, MD-2028, Republic of Moldova \\ ${ }^{3}$ Institute of Physics, Wroclaw University of Technology, \\ Wybrzeze Wyspianskiego 27, 50-370, Wroclaw, Poland
}

(Dated: November 11, 2018)

\begin{abstract}
Raman scattering and photoluminescence (PL) emission are used to investigate a single layer of tungsten disulfide $\left(\mathrm{WS}_{2}\right)$ obtained by exfoliating n-type bulk crystals. Direct gap emission with both neutral and charged exciton recombination is observed in the low temperature PL spectra. The ratio between the trion and exciton emission can be tuned simply by varying the excitation power. Moreover, the intensity of the trion emission can be independently tuned using additional sub band gap laser excitation.
\end{abstract}

\section{INTRODUCTION}

Layered compounds involving transition metals from group VI and chalcogens (the so-called dichalcogenides) are promising candidates for exploring atomically thin structures. The basic building block consists of a monolayer of a transition metal with a chalcogen monolayer above and below. We refer to this chalcogen-metalchalcogen stack as a single layer. All the dichalcogenides have a strong intra layer chalcogen-metal covalent bond while, the layers are weakly held together by van der Waals forces. Nevertheless, the inter layer coupling plays a significant role in determining the band structure. Bulk crystals are semiconductors with an indirect gap in the near infrared spectral range. In contrast, single layer transition metal dichalcogenides such as molybdenum disulfide $\left(\mathrm{MoS}_{2}\right)$, tungsten disulfide $\left(\mathrm{WS}_{2}\right)$ or tungsten diselenide ( $\mathrm{WSe}_{2}$ ) are two dimensional (2D) semiconductors with a direct gap in the visible spectral range ${ }^{[1]}$ The optical response of a single layer of these materials is dominated by excitonic effects; the optical spectrum is characterized by the presence of two low-energy exciton peaks ( $\mathrm{A}$ and $\mathrm{B}$ excitons) that arise from vertical transitions from a spin-orbit-split valence band to a doubly degenerate conduction band at the $\mathrm{K}$ point of the Brillouin zone. $10+12$

The physics of excitons in 2D semiconductors is known to be extremely rich once additional carriers are introduced into the system; the optical spectra consist of emission from both neutral (X) and charged excitons $\left(\mathrm{X}^{ \pm}\right)$. Charged excitons were discovered in II-VI and III-V quantum wells (QWs)! ${ }^{[3 \mid 14}$ In GaAs QWs it has been shown that the ratio between neutral and charged exciton can be tuned by using a back gate, $\frac{14}{16}$ or additional illumination.17]19] In CdTe QWs this ratio can simply be tuned using additional illumination or a change of the excitation power. 20.22 Recently, it has been shown that a gate can be used to tune the carrier density in single layer molybdenum disulfide and molybdenum diselenide $\sqrt{9 \sqrt[23]{23}}$ While in standard semiconductors the dissociation energy of the charged exciton is relatively small
( $\simeq$ few $\mathrm{meV}$ ), in exfoliated dichalcogenides this energy is approximately an order of magnitude larger. Hence, the ability to control the exciton charge state in semiconductor structures which emit light at room temperature and in the visible range, is expected to open many possibilities for optoelectronics applications.

In this paper we show that in a single layer of tungsten disulfide $\left(\mathrm{WS}_{2}\right)$ obtained by the exfoliation of n-type bulk crystals, we observe both charged and neutral exciton recombination in the photoluminescence (PL) emission spectra. Additionally, by simply changing the intensity of the laser excitation, we can tune the ratio between the trion and exciton emission which demonstrates our ability to tune the density of $2 \mathrm{D}$ carriers with light. Moreover, using additional sub band gap laser excitation, the trion emission intensity can be independently tuned. Finally, the temperature dependence of the direct gap of a $\mathrm{WS}_{2}$ single layer is shown to follow the usual behavior for a semiconductor.

\section{EXPERIMENT}

Single and few layer flakes of tungsten disulfide $\left(\mathrm{WS}_{2}\right)$ have been obtained by mechanical exfoliation of bulk $2 \mathrm{H}$ $\mathrm{WS}_{2}$ (the hexagonal $2 \mathrm{H}$-polytype of tungsten disulphide) single crystals grown using chemical vapor transport with Bromine as the transport agent. Samples obtained in this way are naturally n-type ${ }^{24}$ Hall measurements on bulk crystals reveal an electron density $n_{e} \approx 10^{16} \mathrm{~cm}^{-3}$ at room temperature, which decreases rapidly at lower temperatures and is only $\approx 10^{12} \mathrm{~cm}^{-3}$ at $T=140 \mathrm{~K}$. After exfoliation, the $\mathrm{WS}_{2}$ flakes were placed on silicon substrate. Flakes with single layer regions have been identified using optical microscopy, atomic force microscopy (AFM) and Raman spectroscopy. Typical results are presented in Fig 1. Parts of the flake (subsequently referred to as flake 1), which are single layer have a characteristic blue color in the optical microscope image and are also visible in the AFM image. The AFM height profile, measured moving along the green line indicated on the AFM image, is shown Fig 1(c). The $0.6 \mathrm{~nm}$ step in the AFM 

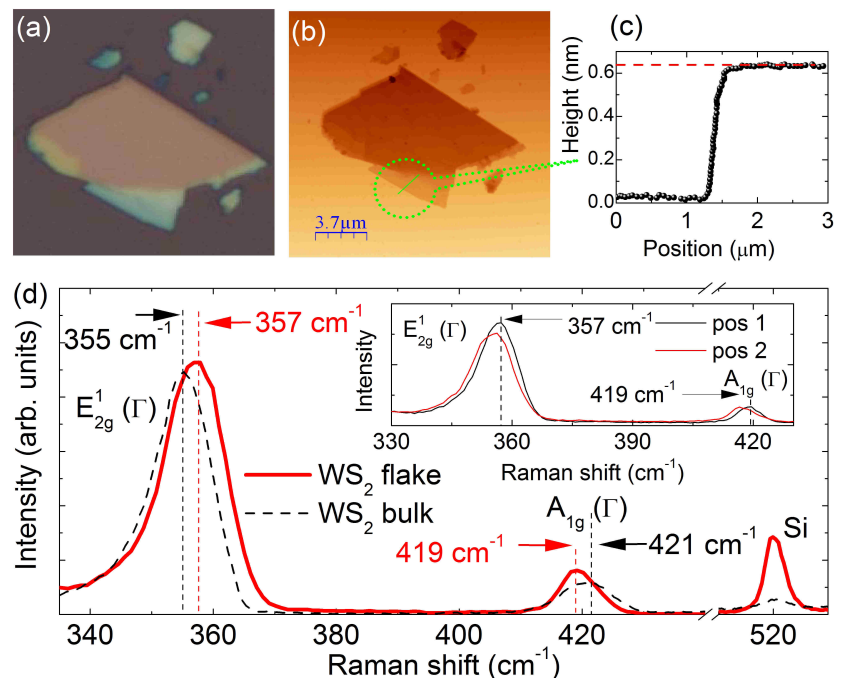

FIG. 1. (color online) (a) 0ptical microscope image and (b) AFM image of the $\mathrm{WS}_{2}$ flake 1. (c) AFM height profile along the path indicated by the green line (d) Raman spectra measured at $\mathrm{T}=300 \mathrm{~K}$ on the single layer area of flake 1 (red line) and bulk $\mathrm{WS}_{2}$ (black dashed line). The inset shows the strain induced shift of Raman spectra taken at two different positions on the single layer area of flake 1 .

height profile corresponds to a single layer $\mathrm{WS}_{2} \cdot \frac{25}{2}$

For the measurements the sample was placed in an optical cryostat mounted on motorized $x-y$ translation stages. Excitation and collection was implemented using an external microscope objective giving a typical spot diameter of $\simeq 1 \mu \mathrm{m}$. The $\mu \mathrm{PL}$ and $\mu$-Raman spectra have been recorded using a spectrometer equipped with a CCD camera and a laser emitting at $532 \mathrm{~nm}$ was used for excitation. For some measurements the sample was simultaneously illuminated by a Ti:Sapphire laser centered at $860 \mathrm{~nm}$, with the laser beams entering collinearly to the microscope objective.

\section{MICRO-RAMAN AND MICRO-PL}

Representative Raman spectra, obtained for a single layer region of flake 1 (red line) and a bulk crystal (black dashed line), are shown in Fig 1(d). The bulk crystal shows two Raman peaks at $355 \mathrm{~cm}^{-1}$ and $421 \mathrm{~cm}^{-1}$ corresponding to the well known active Raman modes $E_{2 g}^{1}(\Gamma)$ and $E_{1 g}(\Gamma)$. However, for the single layer these two peaks shift towards each other by $2 \mathrm{~cm}^{-1}$. These results are consistent with previously reported theoretical calculations and measurements on single layer of tungsten disulfide ${ }^{4}[6]$ Hence, independently of the AFM measurements, the Raman data provides an additional confirmation of the single layer character of the investigated regions of our flakes.

Fig. 2 shows typical $\mu \mathrm{PL}$ spectra measured on a single

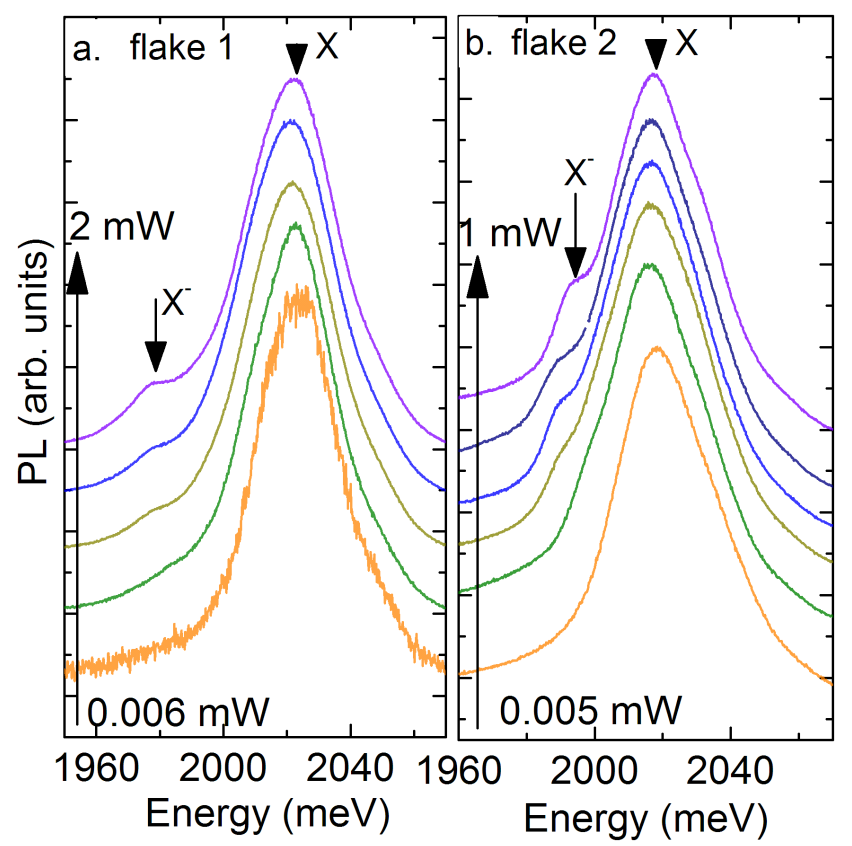

FIG. 2. (color online) Typical $\mu \mathrm{PL}$ spectra measured as a function of the $532 \mathrm{~nm}$ excitation power at $4 \mathrm{~K}$. (a),(b) results obtained for two different flakes. Spectra are offset vertically for clarity

layer region of two different flakes as a function of the excitation power at $T=4 \mathrm{~K}$. Panel (a) corresponds to results obtained on flake 1 . In both cases, at low excitation powers, we observe strong emission around $2 \mathrm{eV}$ which corresponds well with the predicted recombination of the neutral exciton A across the direct gap of single layer $\mathrm{WS}_{2}{ }^{11}$ Moving around the flake the measured emission energy can vary slightly around this value ${ }^{55}$ which we attribute to strain as the Raman spectra show simultaneous small rigid shift of the two Raman peaks with no change in the peak separation (see inset of Fig $1 \mathrm{~d}$ ). This is consistent with the reported influence of the strain on the optical properties of a single layer of $\mathrm{MoS}_{2} \cdot[26 \mid 27$

\section{A. Neutral and charged exciton emission}

Perhaps the most striking feature of the $\mu \mathrm{PL}$ spectra is that we observe a second emission line on the low energy side of the neutral exciton emission at around 1980 meV. This line, which becomes much stronger as the excitation power is increased, is observed in all the flakes we have measured (see e.g. data for flake 2 in Fig 2(b). We will see below that the behavior of the low energy line has all characteristics of a trion; with increasing excitation power, the intensity increases linearly (bi-exciton has quadratic dependence), the binding energy varies linearly, while the ratio of the trion and neutral exciton emission intensity is not constant.

The neutral exciton is the ground state of a charge 
neutral system and trions are only formed in the presence of excess charge which therefore directly controls the intensity of the trion emission. Thus, trion emission is usually not observed in exfoliated samples of $\mathrm{MoS}_{2}$ and $\mathrm{WS}_{2}$ unless a gate is used $\left[\frac{135}{3 \sqrt{3}}\right.$ Gated samples can exhibit an additional line $20-35 \mathrm{meV}$ below the excitonic line which is attributed to the emission from charged excitons $\left.\left(\mathrm{X}^{ \pm}\right) ! 9\right] 23$ Simply by varying the applied gate voltage it is possible to tune the ratio between neutral and charged exciton emission. Hence, in our ungated samples, we attribute the low energy line to emission from charged excitons (trions) which we presume to be negatively charged given the n-type nature of the bulk crystals. The observation of a $X^{-}$requires the presence of excess electrons in the conduction band. In our truly 2D samples internal electric fields cannot spatially separate oppositely charged particles which makes it almost impossible to create excess charge from photo-created electron-hole pairs. However, at low temperature Hall data on the bulk crystals shows that the carriers are frozen out onto the donor levels. Nevertheless, laser illumination, in addition to creating electron-hole pairs, is expected to dynamically photo-ionize carriers trapped on the donors at low temperature, creating a non equilibrium excess electron density in the conduction band. Therefore, we expect that the intensity ratio between neutral and charged exciton can simply be tuned by the varying the power of the laser. To see this more in detail we have analyzed the energy and the intensity of the emission of both $\mathrm{X}$ and $\mathrm{X}^{-}$as a function of the excitation power. The data has been fitted using two gaussian functions to extract the intensity and position of the two lines. The analysis, performed on flakes 1 and 2, is presented in Fig 3(a),(c).

Fig $3(\mathrm{a})$ shows the power dependence of emission energy of the charged and the neutral exciton. With increasing excitation power the neutral and charged exciton emission red shifts towards lower energies with a larger change for the trion. The difference $\Delta E$, between the emission energy of $\mathrm{X}$ and $\mathrm{X}^{-}$, is the dissociation energy of the trion which is presented in Fig $3(\mathrm{~b})$. The trion dissociation energy grows linearly with the excitation power suggesting that the density of the carriers also grows linearly with the laser power: The difference in the energy can be expresses as sum of the binding energy and Fermi energy $\left(E_{X^{-}}+E_{F}\right) \stackrel{20}{20}$ Here binding energy is defined as the dissociation energy in the limit of infinitively small doping $\left(E_{F}=0\right)$ where $E_{X^{-}}$is the energy needed to promote one electron from the trion to the bottom of the conduction band. The Fermi energy increases with increasing power of illumination (photon flux) due to the photo-ionization of donors. The trion dissociation energy (Fermi energy) increases more rapidly with laser power in flake 2 suggesting a larger concentration of donors in this flake. As the carrier concentration increases, the trion dissociation energy increases and we can tune it over approximately $4 \mathrm{meV}$ for both flakes. Such a behavior is also observed in II-VI QWs where the interplay between
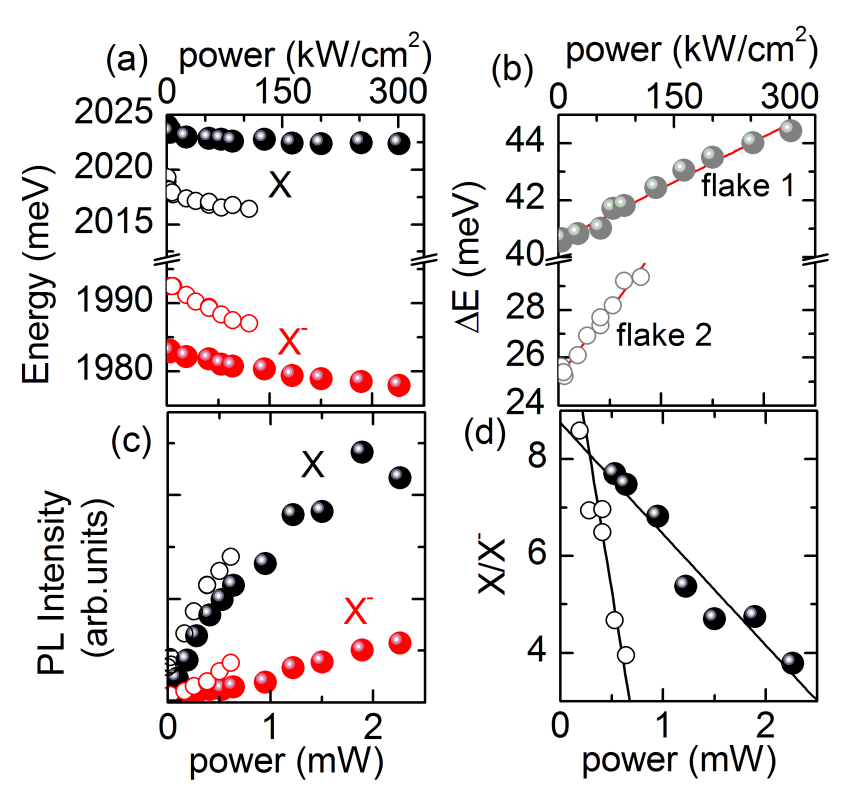

FIG. 3. (color online) (a) The emission energy of the charged and the neutral exciton and their difference (b) as a function of excitation power. (c) integrated intensity of the charged and the neutral exciton and their ratio (d) as a function of excitation power. Data is shown for flake 1 (closed symbols) and flake 2 (open symbols). The lines show the results of a linear fit.

exciton and charged exciton was achieved using external illumination of the sample. $!^{22 \mid 28}$ The enhanced trion binding energy, compared to standard semiconductors, is linked to the true $2 \mathrm{D}$ character of the dichalcogenides and the larger effective mass 12

The calculated integrated intensity of the emission of the charged and neutral exciton, is presented in Fig. 3(c). For both lines the emission intensity increases with increasing power, however, the increase is not the same for the neutral and the charged exciton. To illustrate this the exciton/trion intensity ratio is plotted as a function of laser power in Fig. 3(d). For both flakes the ratio of the integrated intensities decreases with the excitation power by a factor of more than 2 . This is due to increase probability of the creation of the trion with increasing the density of the photo-ionized carriers. However, as the above gap green laser illumination also creates electron hole pairs, the intensity of the neutral exciton emission also increases with laser power. The decrease of the intensity ratio is much faster for the second flake as can also seen directly in the spectra in Fig 2 (b), where the trion line is rapidly becoming stronger with increasing excitation power. Again, the increased sensitivity to laser power is consistent with a larger density of donor impurities in flake 2 . Note, that for both flakes we remain in the linear regime for the trion emission (the exciton emission shows some signs of saturation), so that even at maximum laser power a significant proportion of the 


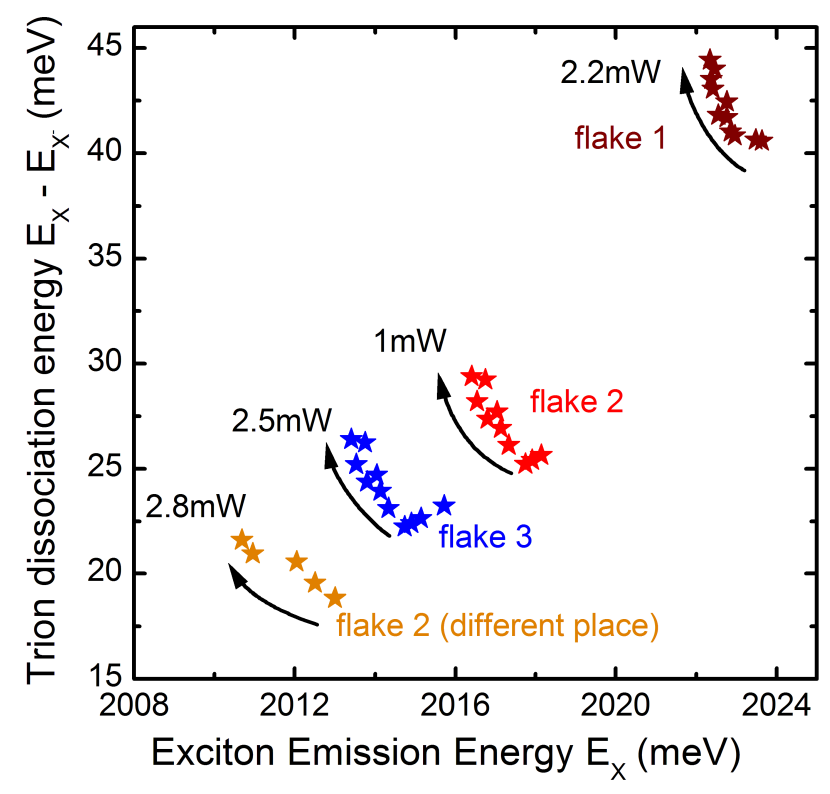

FIG. 4. (color online) The trion dissociation energy $\left(E_{X}-\right.$ $\left.E_{X^{-}}\right)$as a function of the exciton emission energy $\left(E_{X}\right)$ for three different flakes of $\mathrm{WS}_{2}$. For a given flake the exciton emission energy was tuned by varying the Fermi energy via the excitation power used. The arrows show the direction of increasing power from close to zero to the maximum indicated. Green excitation (532nm) was used with the exception of the "flake 2 (different place)" data which corresponds to the two color data in Fig 5

donors are still neutral.

In Fig. 4 we plot the trion dissociation energy $\left(E_{X}-\right.$ $\left.E_{X^{-}}\right)$as a function of the exciton emission energy $\left(E_{X}\right)$ for three different flakes. For a given flake the trion dissociation energy could be tuned over small range by varying the power of the $532 \mathrm{~nm}$ excitation. As previously discussed, with increasing power the trion dissociation energy increases due to the increase in the carrier density (Fermi energy). For all flakes we can tune the trion dissociation energy over a range of $\simeq 4 \mathrm{meV}$. The increase in the trion dissociation energy is accompanied by a small decrease $(1-2 \mathrm{meV})$ of the exciton emission energy which we attribute to an increase in the exciton binding energy due to the reduced screening by trions which are in a spin singlet state and therefore cannot screen effectively due to the Pauli principle. It is important to note that there is a considerable increase $\simeq 20 \mathrm{meV}$ in the trion dissociation energy when going from flake 3 to flake 1 , which is accompanied by a smaller increase $\simeq 10 \mathrm{meV}$ in the exciton emission energy. Within the 2D hydrogen model the exciton binding energy is $E_{B}=4 R_{y}^{*}$, where $R_{y}^{*}$ is the effective Rydberg, and the trion dissociation energy is $\approx R_{y}^{*} / 2=E_{B} / 8{ }^{23}$ From flake to flake the trion dissociation energy varies in the range $20-40 \mathrm{meV}$ corresponding to a change in exciton binding energy within the $2 \mathrm{D}$ hydrogen model from 160 to $360 \mathrm{meV}$. The ob-
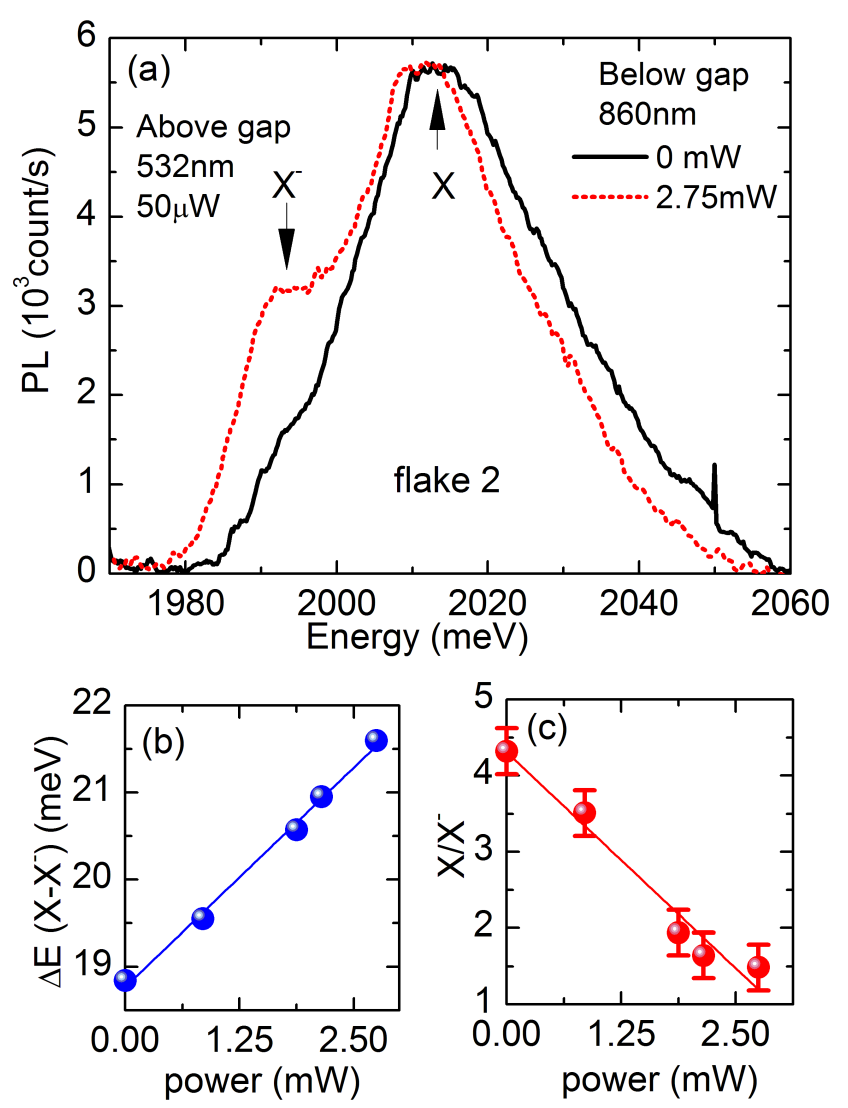

FIG. 5. (color online) (a) Typical $\mu \mathrm{PL}$ spectra measured with and without additional below gap illumination. All spectra are measured with a low constant power of the green $(532 \mathrm{~nm})$ laser $(50 \mu \mathrm{W})$. The difference between emission energy of the neutral and charged exciton (b) as a function of red (860nm) laser power (below gap excitation). The line shows the results of a linear fit. (c) The ratio of the integrated intensity of the charged and the neutral exciton as a function of the red laser power.

served increase in the exciton emission energy is much smaller $(\simeq 10 \mathrm{meV})$ so that the increase in exciton binding energy when going from flake 3 to flake 1 has to be compensated by a similar $(\simeq 170 \mathrm{meV})$ increase in the band gap of the crystal.

\section{B. Independently tuning the trion emission}

In order to tune the trion and neutral exciton emission independently we have performed $\mu \mathrm{PL}$ using two color excitation. A low power $(50 \mu \mathrm{W})$ for the above gap green excitation is used in order to generate a constant density of electron hole pairs together with a small density of photo-ionized electrons. Additional below gap excitation is provided by a laser centered at $860 \mathrm{~nm}(1441$ $\mathrm{meV})$. Photons with this energy, which is well below the gap, do not generate electron-hole pairs. However, their 

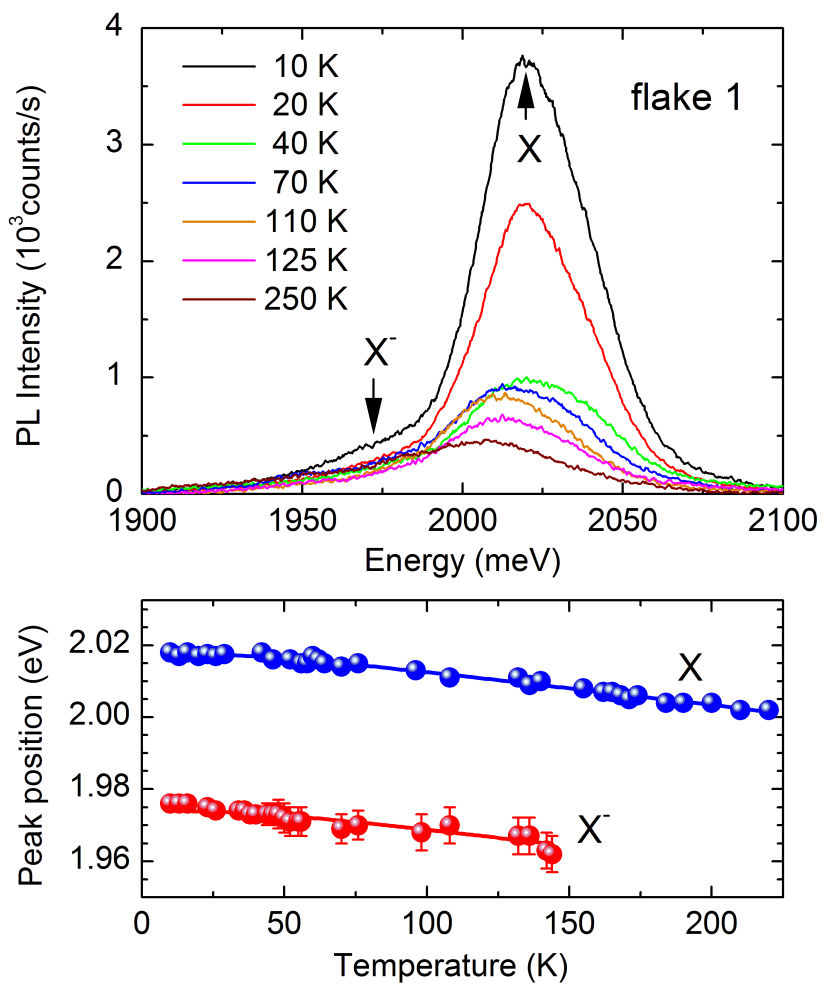

FIG. 6. (color online) (a) Typical $\mu \mathrm{PL}$ spectra measured for different temperatures on flake 1. (b) Emission energy of the neutral and charged exciton as a function of temperature. Solid lines correspond to a fit using equation 1

energy is largely sufficient to photo-ionize electrons from the donor level which is a few hundred meV below the indirect conduction band in bulk crystals. Therefore, we expect that the sub band gap radiation can be used to independently tune the excess density of electrons in the sample.

Representative two color $\mu P L$ spectra measured on flake 2 are presented Fig 5(a). In the absence of below gap illumination, for the low green excitation power chosen, the trion line is very weak. However, when additionally illuminated using a laser at $860 \mathrm{~nm}$, the intensity of the trion emission increases while the (integrated) intensity of the neutral exciton emission decreases. The difference of the emission energy $(\Delta \mathrm{E})$ and ratio of the integrated intensity between neutral and charged exciton determined from the full data set are presented in Fig 5 (b) and (c) respectively. We observe that, as for green excitation, the trion dissociation energy $\Delta \mathrm{E}$ increases linearly with the power of laser and that the intensity ration between neutral and charged exciton also decreases lin- early. This demonstrates that below band gap excitation can be used to vary the electron density in the conduction band and thus to independently tune the intensity of the trion emission.

\section{Temperature dependence of the band gap}

Finally, the temperature dependance of the emission is presented in Fig 6(a). Both exciton lines red shift as the temperatures increases and the charged exciton emission vanishes around $140 \mathrm{~K}$. The energy of the emission, obtained by fitting Gaussians, as a function of the temperature is presented in Fig 6b. Using the standard expression for the temperature dependence of a semiconductor band gap ${ }^{29}$ we can write an expression for the exciton emission energy as a function of temperature,

$$
E(T)=E_{0}-S\langle\hbar \omega\rangle\left[\operatorname{coth}\left(\frac{\langle\hbar \omega\rangle}{2 k_{B} T}\right)-1\right]
$$

where $E_{0}$ is the emission energy at zero temperature, $S$ is a dimensionless coupling constant and $\langle\hbar \omega\rangle$ is the average phonon energy. The best fit to the neutral exciton is obtained with $\left(E_{0}=2.017 \mathrm{eV}, S=0.56\right.$ and $\langle\hbar \omega\rangle \simeq 10.4 \mathrm{meV}$. The charged exciton is well fitted using the same parameters except for the zero temperature emission energy $E_{0}=1.973$. Similar values for the average phonon energy and coupling constant have been obtained for tungsten diselenide. 23 .

\section{CONCLUSION}

Using $\mu \mathrm{PL}$ measurements, we have observed the emission from charged and neutral exciton in an ungated single layer of $\mathrm{WS}_{2}$. The trion emission is closely linked to the n-type nature of our crystals. Using two color $\mu \mathrm{PL}$ with above and below band gap illumination we can independently tune the trion/exciton intensity ratio. The below band gap excitations tunes the excess electron density in the conduction band via the dynamic photoionization of neutral donors. Since the photo-ionization threshold will be similar to the donor binding energy $\simeq 200 \mathrm{meV}$ this provides a possible method for the optical detection of far infrared radiation.

\section{ACKNOWLEDGMENTS}

AAM acknowledges financial support from the French foreign ministry. This work was partially supported by NEXTVALLEY and by ANR project milliPICS.
${ }^{1}$ K. F. Mak, C. Lee, J. Hone, J. Shan, and T. F. Heinz, Phys. Rev. Lett. 105, 136805 (2010)
2 A. Splendiani, L. Sun, Y. Zhang, T. Li, J. Kim, C.-Y. Chim, G. Galli, and F. Wang, Nano Letters 10, 1271 
(2010)

${ }^{3}$ G. Eda, H. Yamaguchi, D. Voiry, T. Fujita, M. Chen, and M. Chhowalla, Nano Letters 11, 5111 (2011).

${ }^{4}$ K. Albe and A. Klein, Phys. Rev. B 66, 073413 (2002)

${ }^{5}$ H. R. Gutiérrez, N. Perea-López, A. L. Elías, A. Berkdemir, B. Wang, R. Lv, F. López-Urías, V. H. Crespi, H. Terrones, and M. Terrones, Nano Letters 0, 0 (2013)

${ }^{\circ}$ W. Zhao, Z. Ghorannevis, L. Chu, M. Toh, C. Kloc, P.-H. Tan, and G. Eda, ACS Nano 7, 791 (2013)

7 Q. H. Wang, K. Kalantar-Zadeh, A. Kis, J. N. Coleman, and M. S. Strano, Nature Nanotechnology 7, 699 (2012)

8 T. Cao, G. Wang, W. Han, H. Ye, C. Zhu, J. Shi, Q. Niu, P. Tan, E. W. B. Liu, and J. Feng, Nature Communications 3, 887 (2012)

${ }^{y}$ K. F. Mak, K. He, C. Lee, G. H. Lee, J. H. T. F. Heinz, and J. Shan, Nature Materials 12, 207 (2013).

10 G. L. Frey, S. Elani, M. Homyonfer, Y. Feldman, and R. Tenne, Phys. Rev. B 57, 6666 (1998)

11 A. Klein, S. Tiefenbacher, V. Eyert, C. Pettenkofer, and W. Jaegermann, Phys. Rev. B 64, 205416 (2001).

12 A. Ramasubramaniam, Phys. Rev. B 86, 115409 (2012)

${ }^{13}$ K. Kheng, R. T. Cox, M. Y. d' Aubigné, F. Bassani, K. Saminadayar, and S. Tatarenko, Phys. Rev. Lett. 71, 1752 (1993).

${ }^{14}$ G. Finkelstein, H. Shtrikman, and I. Bar-Joseph, Phys. Rev. Lett. 74, 976 (1995).

10 J. G. Groshaus, P. Plochocka-Polack, M. Rappaport, V. Umansky, I. Bar-Joseph, B. S. Dennis, L. N. Pfeiffer, K. W. West, Y. Gallais, and A. Pinczuk, Phys. Rev. Lett. 98, 156803 (2007)

Io I. Bar-Joseph, Semiconductor Science and Technology 20, R29 (2005)
17 A. Shields, J. Osborne, M. Simmons, M. Pepper, and D. Ritchie, Phys. Rev. B 52, R5523 (1995).

18 S. Glasberg, G. Finkelstein, H. Shtrikman, and I. BarJoseph, Phys. Rev. B 59, R10425 (1999).

19 J. Jadczak, L. Bryja, A. Wójs, and M. Potemski, Phys. Rev. B 85, 195108 (2012).

${ }_{20}$ V. Huard, R. T. Cox, K. Saminadayar, A. Arnoult, and S. Tatarenko, Phys. Rev. Lett. 84, 187 (2000)

21 A. Esser, E. Runge, R. Zimmermann, and W. Langbein, Phys. Rev. B 62, 8232 (2000)

22 P. Kossacki, J. Cibert, D. Ferrand, Y. Merle d'Aubigné, A. Arnoult, A. Wasiela, S. Tatarenko, and J. A. Gaj, Phys. Rev. B 60, 16018 (1999)

23 J. S. Ross, S. Wu, H. Yu, N. J. Ghimire, A. M. Jones, G. Aivazian, J. Yan, D. G. Mandrus, D. Xiao, and X. Yao, Wang Xu, Nature Communications 4, 1474 (2013)

${ }^{24}$ S. H. El-Mahalawy and B. L. Evans, Phys. Status Solidi B 79, $713(1977)$

${ }^{25}$ W. Schutte, J. D. Boer, and F. Jellinek, Journal of Solid State Chemistry 70, 207 (1987)

${ }^{20}$ H. J. Conley, B. Wang, J. I. Ziegler, R. F. H. Jr., S. T. Pantelides, and K. I. Bolotin, arXiv:1305.3880v1 (2013).

27 C. Rice, R. J. Young, R. Zan, U. Bangert, D. Wolverson, T. Georgiou, R. Jalil, and K. S. Novoselov, Phys. Rev. B 87, 081307 (2013)

${ }_{28}$ P. Kossacki, H. Boukari, M. Bertolini, D. Ferrand, J. Cibert, S. Tatarenko, J. A. Gaj, B. Deveaud, V. Ciulin, and M. Potemski, Phys. Rev. B 70, 195337 (2004).

29 K. P. O'Donnell and X. Chen, Applied Physics Letters 58, 2924 (1991). 